Mots. Les langages du politique

\title{
Les discours d'investiture des Premiers ministres portugais (2002-2011). Comparaison gauche/droite
}

Michèle Monte

\section{(2) OpenEdition \\ Journals}

Édition électronique

URL : https://journals.openedition.org/mots/21096

DOI : $10.4000 /$ mots. 21096

ISSN : 1960-6001

Éditeur

ENS Éditions

Édition imprimée

Date de publication : 22 avril 2013

Pagination : 13-30

ISBN : 978-2-84788-393-0

ISSN : 0243-6450

Référence électronique

Michèle Monte, «Les discours d'investiture des Premiers ministres portugais (2002-2011)

Comparaison gauche/droite », Mots. Les langages du politique [En ligne], 101 | 2013, mis en ligne le 22

avril 2015, consulté le 23 avril 2022. URL : http://journals.openedition.org/mots/21096 ; DOI : https:// doi.org/10.4000/mots. 21096

\section{(C) ENS Éditions}




\section{Les discours d'investiture des Premiers ministres portugais (2002-2011). Comparaison gauche/droite}

Le système politique portugais est de type parlementaire. Le président de la République est chargé par la Constitution de désigner la personne qui devra former le gouvernement ${ }^{1}$. Cette personne est généralement le chef du parti ou de l'alliance majoritaire à l'Assemblée. Le Premier ministre est investi par le président de la République et prononce devant lui son discours d'investiture. Il ne s'agit pas dans ce discours de présenter un programme (ce sera fait devant l'Assemblée de la République, qui peut le rejeter), mais de répondre au président qui rappelle les grands principes et exprime son avis sur les urgences de l'heure. L'enjeu est d'acquérir ou de confirmer une stature de chef de gouvernement et de préciser les orientations générales qui vont être suivies. L'auditoire du discours, au-delà du président de la République, des présidents de l'Assemblée, de la Cour suprême de justice, du Tribunal constitutionnel, des ministres, des ambassadeurs et autres invités, est bien sûr l'ensemble des citoyens portugais. La cérémonie se tient dans le Palais de l'Ajuda, ancien palais royal réservé aux cérémonies officielles.

Cinq Premiers ministres se sont succédé de 2002 à 2011 au gré des alternances issues des différentes consultations électorales. En 2002, le chef du Parti social-démocrate (centre droit), José Manuel Durão Barroso, succède au socialiste António Guterres qui démissionne après une série de difficultés, ce qui donne lieu à des élections anticipées en mars. Barroso dirige une coalition PSD-PP (Partido popular). Nommé président de la Commission européenne en 2004, il doit démissionner et c'est un membre de son parti qui lui succède, Pedro Santana Lopes. Mais il ne reste au pouvoir que 168 jours en raison d'une

Je tiens à exprimer ma reconnaissance à Maria Aldina Ferreira Marques sans qui je n'aurais pu mener à bien la collecte du corpus.

1. Article 187 de la Constitution : «O Primeiro-Ministro é nomeado pelo Presidente da República, ouvidos os partidos representados na Assembleia da República e tendo em conta os resultados eleitorais. » ("Le Premier ministre est nommé par le président de la République, après consultation des partis représentés à l'Assemblée de la République et en prenant en compte les résultats électoraux.»)

Université de Toulon, Babel EA 2649

michele.monte@univ-tln.fr 
série de scandales qui conduisent le président de la République à dissoudre l'Assemblée fin 2004, un an et demi avant la fin de la législature². Les élections anticipées de 2005 donnent la majorité absolue au Parti socialiste et c'est son chef, José Sócrates, qui devient Premier ministre. À la fin de la législature, en 2009, Sócrates est réélu mais avec la majorité relative seulement, ce qui réduit sa marge de manœuvre à l'Assemblée. En 2011, alors que la crise financière touche le Portugal de plein fouet, Sócrates ne peut obtenir un vote favorable sur son programme de redressement économique, ce qui entraîne des élections législatives anticipées et l'arrivée au pouvoir de Pedro Passos Coelho, du PSD. Je dispose donc pour la période de cinq discours d'investiture, trois prononcés par des Premiers ministres issus du PSD et deux par un Premier ministre issu du PS3. Cette période est par ailleurs caractérisée par la montée des difficultés financières du Portugal, dues à l'explosion de la dette et à la spéculation des banques contre la dette souveraine portugaise.

À travers l'étude du vocabulaire utilisé dans ce discours et des positionnements assumés par les Premiers ministres successifs, je me propose de dégager ressemblances et différences dans la façon de représenter les atouts et les difficultés du Portugal, ainsi que les causes et les remèdes de la crise et l'action du gouvernement. L'objectif final sera de m'interroger sur l'efficacité argumentative de tels discours auprès des citoyens.

\section{Nation, pays, Portugal, peuple, Portugais : de fortes convergences}

On peut s'attendre aux désignations suivantes pour le pays : Portugal, o País, o nosso País, a nação, a pátria. Par ailleurs, l'entité nationale peut être mise en relation avec d'autres entités à l'intérieur et à l'extérieur des frontières; à l'intérieur, on trouve o povo, os Portugueses, os cidadãos, et à l'extérieur, a Europa, os Paises de língua oficial portuguesa4 (PLOP dans le tableau). La communauté internationale est absente. On trouvera en annexe (tableau 1) le détail des résultats5.

Il semble, au vu de ce corpus certes restreint, que l'usage portugais ne diffère pas de celui étudié en France par Paul Bacot (1996) dans les professions

2. Sur cet épisode, très intéressant pour les relations présidence/gouvernement, voir Jalali 2007, p. 254 et suiv.

3. Les discours seront désignés par les deux premières lettres du dernier nom de celui qui les prononce, suivies de I ou II pour Sócrates. Je rappelle la date ici même de chaque discours mais ne la reprendrai pas ensuite : BA (2002), LO (2004), SO I (2005), SO II (2009), CO (2011). On trouvera l'original portugais dans le corps du discours et la traduction en note.

4. Successivement: le Portugal, notre pays ou le pays, la nation, la patrie, le peuple, les Portugais, les citoyens, l'Europe et les Pays de langue officielle portugaise.

5. Le corpus a été traité avec le logiciel TXM développé par l'équipe LINCOBATO du laboratoire ICAR. 
de foi des candidats ${ }^{6}$. Les désignations dominantes sont celles qui sont le plus consensuelles, les plus neutres, c'est-à-dire Portugal et le pays ou notre pays (7 occurrences avec notre, et 35 avec majuscule)7 qui sont respectivement le deuxième et le troisième substantif les plus utilisés de tout le lexique. Conformément à ce que laisse attendre l'étude lexicologique menée par Sylvianne Rémi-Giraud (1996), le mot le plus chargé d'affect, à savoir patrie, et le mot qui possède le plus fort contenu politique, à savoir nation, sont peu utilisés. Sócrates utilise deux fois patrie dans la conclusion de son deuxième discours, une fois dans une citation du poète national Camões et une fois en association avec la République :

«Esta é a ditosa pátria minha amada». Inspiro-me em Camões para terminar. Aqui estamos. Para servir a República. Para servir os portugueses. Para servir a Pátria que, tal como Camões, todos amamos. ${ }^{8}$

C'est le seul discours où la République (fondée en 1910) apparait ailleurs que dans les collocations président de la République et Assemblée de la République (désignation portugaise de l'Assemblée législative) : contrairement à la France, la notion de République est rarement mobilisée par les hommes politiques portugais. On peut penser que cette apparition dans le discours de 2009 est due à l'approche du centenaire de sa naissance. Pátria et República concourent ici à un effet de pathos en renouant avec des références historiques anciennes.

La nation est invoquée dans deux contextes très différents : Barroso et Santana Lopes en font la source de la légitimité gouvernementale et, partant, de l'obligation d'être au service de tous', alors que Passos Coelho la présente "affligée par des problèmes » qu'il s'agit de résoudre conjointement avec les «dilemmes que rencontre l'Union [européenne]». Il la range ainsi implicitement parmi les autres nations européennes avec lesquelles elle doit renforcer sa coopération. Dans les deux cas c'est l'entité politique qui est visée, mais une fois dans son unité interne, l'autre fois dans ses rapports avec l'extérieur. C'est surtout le premier emploi qui s'actualise pour l'adjectif nacional, associé

6. L'observation du tableau proposé par Maurice Tournier (1996, p. 67-68) à partir de quelques corpus politiques français offre matière à des comparaisons intéressantes que je ne peux développerici.

7. L'absence de majuscule dans 8 cas sur 43 correspond presque toujours à un emploi prédicatif: "Nous voulons un pays plus ouvert, plus solidaire».

8. «Voici mon heureuse patrie bien-aimée». Je m’inspire de Camões pour terminer. « Nous voici. Pour servir la République. Pour servir les Portugais. Pour servir la Patrie que tous, à l'instar de Camões, nous aimons.»

9. "Este Governo, como deve ser em democracia, assenta numa base partidária. Mas quero que fique claro que a partir deste momento não éo Governo de um ou de outro partido. Éo Governo da Nação. » (BA) ("Ce gouvernement, comme il convient en démocratie, émane des partis majoritaires. Mais je veux qu'il soit bien clair qu'à partir de maintenant, il n'est pas le gouvernement de tel ou tel parti. Il est le gouvernement de la Nation. ») 
à credibilidade (BA) et egoismos (S0) mais surtout à consenso, pacto, coesão, comunidade, interesse, recursos ${ }^{10}$. Cependant, le fait que les Premiers ministres préfèrent tous largement Portugal et pays à nation montre que l'entité qu'ils envisagent est naturalisée sur une base territoriale plus qu'envisagée comme le fruit d'un contrat entre citoyens. La fréquence bien moindre des mots peuple et citoyens par rapport au pluriel les Portugais confirme cette tendance. Certes, l'apparition de peuple est liée à l'expression de la volonté populaire dans la moitié des occurrences, mais celle-ci a surtout pour rôle de légitimer le gouvernement qui est relié au peuple par un contrat de confiance : le peuple s'est exprimé (SO 2005), il confère au gouvernement son mandat (LO), il a élu le gouvernement et doit pouvoir compter sur lui (CO). Le discours d'investiture apparait comme un moment privilégié pour redire ce lien qui unit les citoyens à leur gouvernement, et que résume le mot responsabilidade appliqué la plupart du temps au gouvernement, mais parfois aussi au peuple ou aux autres institutions ( forces parlementaires » pour SO 2009, "partenaires sociaux» pour CO). Sócrates I est celui qui insiste le plus sur les droits et devoirs des citoyens, mot qu'il emploie nettement plus que les autres Premiers ministres. Quant à Santana Lopes, il fait un usage spécifique et abondant du mot peuple : il utilise six fois l'expression como povo ("en tant que peuple») pour désigner l'entité humaine collective qui rassemble tous les citoyens. C'est le peuple qui fait face aux difficultés, qui peut les surmonter «avec des principes, des règles et du courage », qui doit se faire comprendre à l'extérieur, atteindre ses objectifs. Massés au milieu et à la toute fin du discours, ces emplois sont associés à l'évocation de l'histoire du Portugal :

Daqui [« notre aventure sur les mers de Chine » référée plus haut] retiramos orgul ho como nação, mas retiramos, ao mesmo tempo, forças novas que nos fazem, como povo, sentir capaz de vencer problemas e desafios. ${ }^{11}$

O caminho é estreito, mas seremos capazes. Seremos capazes como Governo porque seremos capazes como povo. ${ }^{12}$

Comme on le voit dans le deuxième exemple, l'emploi de ce mot associé au pronom personnel nous assure la fusion entre le gouvernement et les citoyens que les syntagmes le pays, les citoyens, les Portugais ne permettent pas.

Si l'on s'intéresse à présent au voisinage des mots Portugal et pays, en essayant de saisir leurs éventuelles spécificités, les deux mots semblent a priori relativement interchangeables. Tous deux ont des «problèmes à affronter», des «défis à relever». On observe toutefois que Portugal apparait dans

10. Respectivement consensus, pacte, cohésion, communauté, intérêt, ressources.

11. "C'est de cela que nous pouvons nous enorgueillir comme nation, mais nous en retirons aussi des forces nouvelles qui nous font, comme peuple, nous sentir capables de vaincre les problèmes et les défis. ». L'adjectif capable est associé trois fois à peuple.

12. "Le chemin est étroit, mais nous aurons les capacités. Nous aurons les capacités comme gouvernement parce que nous les aurons comme peuple.» 
des phrases plus solennelles, en ouverture et surtout en clôture des discours : cela est dû notamment au fait que les relations extérieures du pays sont évoquées en dernière partie et que, dans ces emplois, Portugal est systématiquement préféré à pays, comme le montre cet énoncé parmi d’autres : «Portugal tem hoje uma visibilidade e uma responsabilidade acrescidas no concerto das nações. \13 (SO 2009). Par ailleurs Portugal est préféré dans les énoncés où la personnification est plus sensible, par exemple comme complément du verbe servir ou sujet du verbe savoir :

Portugal jamais poderá regressar à ilusão de que a divida em espiral alimenta crescimento. Portugal sabe por experiência própria que a embriaguez da dívida se limita a encenar um falso e curto bem-estar até ao dia em que chega a factura e o colapso. ${ }^{14}(\mathrm{CO})$

À l'inverse, les mots situation ou modernisation accompagnent pays qui se prête davantage à des développements descriptifs en raison de son noyau sémantique ancré dans un territoire. Face au Portugal «qui s'est appauvri » mais qui «ne peut échouer» (BA), on trouve ainsi le souhait d'un «pays plus ouvert» (CO). Cependant, les métaphores du rétablissement et du retard à rattraper sont utilisées par BA et LO avec pays et des énoncés ou syntagmes comme «le redressement économique que le pays est en train d'accomplir» (LO), « le pays a besoin de l'apport de tous », « les défis que le pays doit relever» (SO 2009) montrent qu'une certaine personnification est possible avec pays.

\section{Relations extérieures : « option européenne » et "vocation atlantique "}

Sur le plan extérieur, l'ancrage européen est réaffirmé par tous les Premiers ministres. Pour Barroso, l’objectif premier est de «retrouver la convergence avec l'Union européenne» par "un assainissement des comptes publics», ainsi pourra-t-on «replacer le Portugal au centre de la construction européenne »; plagiant Térence, il affirme que «rien de ce qui est européen ne nous est étranger». Pour Santana Lopes, «l'Europe n'est pas pour le Portugal une contrainte; en faire partie, participer pleinement à ce projet encore en gestation est une option que nous avons prise librement »; pour Sócrates I, il faut « réaffirm[er] la centralité de notre option européenne et assur[er] une participation pleine et active du Portugal dans la construction de l’Europe».

13. «Le Portugal détient aujourd'hui une visibilité et une responsabilité accrues dans le concert des nations.»

14. «Le Portugal ne pourra jamais revenir à l'illusion que la spirale de la dette alimente la croissance. Le Portugal sait d'expérience que l'ivresse de la dette se borne à mettre en scène un bienêtre éphémère et fallacieux jusqu'au jour où viennent la facture et l'effondrement. » 
L'affirmation traditionnelle des liens avec les pays lusophones vient en second et apparait plus comme un passage obligé pour réaffirmer la dimension internationale d'un «pays géographiquement petit» (LO) ou, plus nettement encore, d'un «petit pays» (SO 2005), que comme un véritable projet politique. Il existe néanmoins deux différences intéressantes : seuls Santana Lopes et Sócrates I évoquent la place du pays dans l'OTAN et ses «relations d'amitié et de coopération réciproque avec les États-Unis d'Amérique» (SO). Si ces affirmations n'étonnent pas chez le Premier ministre du PSD, qui insiste ensuite longuement sur « notre attachement aux valeurs qui sont la base de la civilisation occidentale», elles expriment peut-être chez Sócrates le désir de rassurer le puissant allié au moment où la gauche revient au pouvoir. Quant à l'expression «vocation atlantique » qu'on trouve chez Sócrates I et II et Passos Coelho, elle peut recouvrir aussi bien l'ouverture ancienne du Portugal vers l'outremer que ses liens avec la superpuissance américaine, comme le dit explicitement Santana Lopes à propos de la «dimension atlantique» du Portugal.

En ce qui concerne les relations avec l'Union européenne, on note des différences assez sensibles dans les formulations: Barroso met l'accent sur les engagements vis-à-vis de l'Europe ; Santana Lopes affirme que l'appartenance à l'Union est «la voie qui sert le mieux les intérêts du Portugal et des Portugais» et insiste sur l'égalité des droits et devoirs de tous les membres; Sócrates I évoque «le projet européen d'une citoyenneté supranationale, capable de dépasser les égoïsmes nationaux», mais en 2009, les mots Europe et européen sont quasiment absents de Sócrates II - sorte de déni désespéré de la réalité du pays en 2009? - et leur présence est euphémisée chez Coelho qui évoque l'aide autrefois apportée par l'Europe ${ }^{15}$ et un travail «d'égal à égal» avec les «partenaires», pour terminer de façon plutôt vague sur la «complexité des contraintes européennes » alors même que les exigences de la «troïka »16 s'imposent à la politique portugaise. Le respect des engagements européens est envisagé au début de la décennie comme un facteur de progrès et aussi comme une question d'honneur, il est ensuite mis au second plan au moment même où il conditionne complètement les choix politiques.

\section{Difficultés et perspectives : une commune euphémisation}

Lorsque l'on étudie la schématisation discursive (Grize, 1990, p. 35) de la situation qu'affronte le Portugal, on constate sans grande surprise que les discours

15. Il y a là une allusion au passé récent : s'agit-il de l'entrée dans l'UE en 1986 ou d'un appui de l'Europe de l'Ouest au moment où, après la Révolution des Fillets, certains craignaient que le Portugal ne bascule vers un régime communiste? Les deux semblent possibles.

16. Telle est l'appellation dans les médias portugais de la Mission UE/BCE/FMI qui a imposé un rigoureux plan d'austérité au Portugal. 
d'une part recensent des difficultés ou problèmes, d'autre part s'efforcent de proposer des remèdes ou des perspectives, mais le poids donné à tel ou tel mot diffère sensiblement d'un discours à l'autre, comme on peut le voir dans le tableau 2 en annexe, où les mots étudiés sont donnés en portugais.

En ce qui concerne les difficultés, deux points me semblent à relever : l'emploi contrasté de crise et la tendance à l'euphémisation. Seuls Barroso et Sócrates II emploient crise de façon significative. Pour Barroso, le mot réfère dans 2 cas sur 5 à la démission du précédent Premier ministre, dans 2 autres cas à l'état des finances publiques, et la crise justifie le changement de cap voulu par les électeurs et présenté comme un remède : "La crise va laisser place à l'espérance », telle est la conclusion du discours où le mot amalgame les deux sens antérieurs. Pour Sócrates II, il s'agit de la "crise économique mondiale» et 4 fois sur 6, crise est précédé d'un mot tel que combattre, combat, vaincre. Sócrates prend appui sur la crise pour justifier son programme d'innovation et de modernisation. L'absence du mot en 2005 ne signifie pas que la thématique est absente, puisque Sócrates I parle de la « récession », mais il préfère à ce moment-là se centrer sur l'aggravation des inégalités sociales qu'elle entraîne. Si Santana Lopes et Passos Coelho emploient peu le mot, lui préférant problème(s), c'est qu'ils ne veulent pas, au nom d'une crise mondiale, exonérer de leurs responsabilités le pays ou les gouvernements PS qui les ont précédés. Il est significatif que le pays dans son entier soit presque toujours associé au mot problème : nos problèmes, problèmes nationaux, problèmes qui nous affligent comme nation, problèmes qui nous frappent, problèmes que nous avons créés. Santana Lopes recourt également à difficulté(s) mais fait surtout un usage plus abondant que les autres Premiers ministres du mot effort(s) en se référant 4 fois aux efforts du gouvernement antérieur, 1 fois aux familles et entreprises, mais 3 fois à des entités collectives («notre génération », «le Portugal», «tous») et 2 fois sans qu'un agent soit exprimé. Cette insistance tend à donner une vision psychologique de la situation de crise et se substitue à l'énoncé de priorités, comme nous le verrons plus bas.

Par ailleurs, même si l'on note une augmentation en 2011 des mots du champ lexical des difficultés, on note qu'aucun des Premiers ministres ne dramatise la situation. Ils s'efforcent tous de rassurer le pays sur la capacité du gouvernement à résoudre les problèmes tout en faisant appel aux efforts de tous, même si Sócrates est le seul à utiliser expressément les mots mobiliser et mobilisation. Barroso affirme que «le Portugal ne peut échouer», et cet énoncé est repris tel quel en clôture de son discours par Passos Coelho qui ajoute : "Je sais que le Portugal n'échouera pas». Sócrates I se propose de «transformer le Portugal des fatalités» en «Portugal des opportunités», et Sócrates II déclare : "Tel est le Portugal que nous voulons : un pays moderne, ouvert au monde, qui valorise les personnes, leurs compétences, leurs énergies». On notera que Santana Lopes et Sócrates se rejoignent pour l'emploi fréquent de 
défi mais que Sócrates préfère y associer futur là où son prédécesseur répétait (10 occurrences) : «nous sommes capables »17.

Si l'on envisage maintenant les remèdes, on constate, par rapport à la France où il semble être le maître mot des discours politiques, une quasi-absence du mot réforme. Le mot progrès, quant à lui, figure encore en ces temps difficiles dans 4 discours sur 5 , certes à une place modeste, mais la croyance au progrès reste très présente dans le discours de Sócrates où elle se manifeste en 2005 par le suremploi des mots innovation, innover, novateur et en 2009 par celui de moderne, moderniser et modernisation. Là où Barroso invite à l'espérance en se situant sur un registre plutôt moral, Sócrates parie sur les changements technologiques et le développement des connaissances. Santana Lopes insiste pour sa part sur la question de l'image du Portugal : le mot est absent des quatre autres discours mais figure 6 fois dans le sien, concentré sur trois paragraphes et présenté comme un remède aux difficultés du pays :

Bem sabemos que a imagem que mais importa ter no mundo da competição global em que vivemos é a da qualidade. [...] Há, no entanto, uma imagem que Portugal pode projectar no Mundo : a de país seguro. [...] Ter, no mundo de hoje, a imagem de um destino seguro, para além de um bem em si mesmo é naturalmente dispor de uma enorme vantagem competitiva. ${ }^{18}$

Quant à la forte fréquence du mot changement dans le discours de Coelho, elle se réfère non pas à des changements à introduire mais à celui apporté par le vote des Portugais qui mettent fin à six ans de gouvernement socialiste. La réponse de Coelho à la crise se marque par le suremploi spectaculaire de ouvert/ouverture, dont je traiterai dans la partie suivante.

L'étude lexicale montre ainsi des différences importantes dans le détail mais une présentation globale similaire. Dans chacun des discours, on trouve l'énoncé de difficultés suivi de la conviction nettement affirmée que les solutions sont à portée de main, mais qu'elles nécessitent l'engagement de tous. Les difficultés sont désignées de façon différente mais rapportées à une conjoncture globale de compétitivité mondiale féroce qui apparait comme indépassable. Les priorités énoncées offrent, quant à elles, plus de différences.

17. «Não temos de ser um povo de segunda no contexto europeu porque somos tão capazes como os melhores. » («Nous n'avons pas à être un peuple de deuxième catégorie dans le contexte européen parce que nous sommes aussi capables que les meilleurs. »)

18. Nous savons bien que l'image qui importe le plus dans le monde de la compétition globale où nous vivons est celle de la qualité. [...] Il y a, cependant, une image que le Portugal peut répandre dans le monde : celle d'un pays sûr. [...] Avoir, dans le monde d'aujourd'hui, l'image d'un destin sûr, non seulement est un bien en soi mais c'est aussi, naturellement, disposer d'un énorme avantage compétitif. 


\section{Priorités : des différences significatives}

Presque tous les orateurs soulignent leurs priorités et quatre discours sur cinq sont très structurés. Seul le discours de Santana Lopes, au demeurant le plus long, présente un plan moins apparent. Après avoir affirmé qu'il respecterait la Constitution et fonderait son action sur la «démocratie» et le «développement », il évoque le rôle de l’État, la nécessité du dialogue, «la culture de l'action » qui caractérise son gouvernement, et l'histoire du Portugal qui doit permettre d' "avancer parce que notre retard n'est pas une fatalité de l'Histoire». Il en vient ensuite aux difficultés : "crise des finances publiques », excessif "endettement des familles et des entreprises» et "perte de compétitivité de nos produits». Il développe ensuite sa "stratégie» de «réponse » à ces trois "contraintes» avant d'exprimer longuement sa vision de la politique extérieure du Portugal. C'est sur la partie "stratégie» que portera la comparaison avec les autres discours. Barroso distingue des «objectifs supérieurs » (points 3, 4, 5 du tableau 3 en annexe) et des mesures «à prendre sur-le-champ » $(1,2)$ qui «ne constituent pas une fin en soi mais un moyen pour la réalisation » de ces objectifs. Il énonce d'abord les secondes pour revenir aux premiers ensuite. Sócrates I dessine d'abord "un cadre de principes et valeurs structurants» puis énonce «trois grandes priorités». Sócrates II formule «trois priorités» et Passos Coelho trois «tâches prioritaires». Il est donc facile à l'analyste de confronter les objectifs assignés à l'action gouvernementale (voir en annexe).

Il y a une nette différence entre les discours des Premiers ministres PSD et ceux du Premier ministre PS : même si la préoccupation de bonne gestion budgétaire n'est pas absente des propos de Sócrates ${ }^{19}$, il met la croissance et l'emploi au centre de ses objectifs ${ }^{20}$ avec cependant un changement important de 2005 à 2009 qui reflète peut-être à la fois l'aggravation de la crise et son euphémisation dans le discours. On trouve en effet six occurrences pour crescimento («croissance») en 2005 et une seule en 2009. Quant au chômage, c'est une thématique forte en 2005 (6 occurrences pour desemprego/desempregado), alors qu'il disparait au profit de l'emploi en 2009 (5 occurrences d'emprego). Sur ce plan, Sócrates II et Coelho ne se distinguent pas, préférant parler de «création d'emploi » que de diminution du chômage, peut-être par peur des promesses non tenues. En revanche Sócrates est le seul à parler de la réduction

19. «Não esqueceremos, também, que finanças públicas sãs são condição necessária - não suficiente, mas necessária - para o desenvolvimento económico e para a criação de emprego. » (SO 2005) («Nous n'oublierons pas, non plus, que des finances publiques saines sont une condition nécessaire - non suffisante, mais nécessaire - pour le développement économique et pour la création d'emplois. »)

20. «É no crescimento económico e no emprego que concentraremos o essencial das nossas energias. » (SO 2009) ("C'est sur la croissance économique et l'emploi que nous concentrerons l'essentiel de nos énergies.») 
des inégalités : il emploie 6 fois le mot égalité (toujours au singulier) et 4 fois le mot inégalités (toujours au pluriel), absents des autres discours ${ }^{21}$. Coelho préfère parler, lui, d'un « Programme d'urgence sociale, orienté vers les personnes qui souffrent des plus fortes carences et vers ceux qui sont les plus sévèrement atteints ». On notera la préférence accordée à un vocabulaire médical qui fait de la crise une maladie. L'emploi du mot justice est lui aussi clivant mais isole Coelho : alors que Barroso, Santana Lopes et Sócrates emploient le syntagme justice sociale une ou deux fois dans leur discours, Coelho, lui, emploie le syntagme «justice intergénérationnelle» et parle surtout ( 7 de ses 8 occurrences de justice) des réformes à apporter au système judiciaire.

Les spécificités qui se dessinent semblent dues pour partie à l'appartenance politique des locuteurs et pour partie à l'évolution de la conjoncture. Barroso insiste sur le lien entre équilibre budgétaire et crédibilité ( 4 des 5 occurrences du mot dans le corpus) dans un contexte d'adhésion récente à l'euro, qui, rappelle-t-il, a fait l'objet d'un «consensus national». Santana Lopes et Sócrates sont tous deux friands des mots investissement (respectivement 6 et 4 occurrences) et développement ( 9 et 5 occurrences). Le premier associe développement et progrès social alors que le second espère rendre l'économie plus compétitive par l'amélioration de la scolarité 22 et par l'innovation technologique. Il affirme ainsi en 2009 vouloir " positionner l'économie portugaise sur la nouvelle frontière technologique de la recherche et du développement».

Coelho insiste, lui, d'une part sur le «tarissement» (estancamento) de la dette, d'autre part sur la "dévalorisation fiscale», expression entre guillemets, qui permettront de « retrouver la compétitivité externe » et de «créer de l'emploi en diminuant la pénalisation fiscale du travail». Si l'emploi des mots équilibre, rigueur ${ }^{23}$, assainissement des comptes, compétitivité, transparence, n'est pas propre à tel ou tel locuteur, les lexèmes associés dessinent clairement un discours économique libéral dans le cas de Coelho, un discours économique interventionniste dans le cas de Sócrates et un discours centré sur l'image du pays à l'extérieur dans le cas de Barroso. Quant à Santana Lopes, il se distingue par l'association de l'interventionnisme de l'État et de l'appel aux entrepreneurs : il totalise 10 des 23 occurrences des mots entreprise, entrepreneurs et entrepreneurial.

Le lexème le plus intéressant en termes de spécificité est celui d'abertura («ouverture»), dont Coelho fait le maître mot de son programme, et qui, de même que l'adjectif dont il dérive, change au fil du discours. Alors qu'au tout

21. Santana Lopes parle une fois d'égalité entre les citoyens et les États-membres de l'Union européenne.

22. Sócrates emploie 6 fois éducation et 3 fois école, Santana Lopes une fois éducation et une fois éduquer, ils sont les seuls à le faire et à recourir aussi au thème de l'innovation technologique. Il y a là sans doute une influence de la «stratégie de Lisbonne » qui appelait en 2000 à développer «l'économie de la connaissance» pour améliorer la compétitivité européenne.

23. C'est Sócrates qui l'utilise le plus (3 sur 5 des occurrences). 
début, l'ouverture caractérise les relations entre le gouvernement et le peuple, elle exprime ensuite la croyance dans les vertus de la libre entreprise et de l'initiative individuelle, pour finir p. 4 (après l'énoncé p. 3 des trois programmes prioritaires) par se restreindre à la seule ouverture à la concurrence et aux investissements. L'orientation libérale des gouvernements du PSD se manifeste aussi dans leurs emplois spécifiques des lexèmes État, société et démocratie.

\section{État, société et démocratie : des relations contrastées}

Les différences entre discours de gauche et discours de droite sont particulièrement nettes en ce qui concerne ces trois lexèmes, comme on le voit dans l'annexe III le tableau 4. La fréquence du mot gouvernement - c'est le premier des mots pleins du lexique - tient aux conditions d'énonciation, mais il faut noter que Coelho préfère utiliser massivement la première personne du pluriel plutôt que le gouvernement pour présenter ses orientations. Le rôle attribué à l'État reflète directement les positions politiques des locuteurs, sauf pour Santana Lopes. Barroso et surtout Coelho incarnent la vision libérale de l'État, alors que Santana Lopes affirme à la fois le droit des citoyens contre l'État et le rôle éminent de celui-ci au service de tous, et que Sócrates voit dans l'État le défenseur des faibles. Voyons cela plus en détail.

Pour Barroso, l’État incarne la puissance publique à l'intérieur comme à l'extérieur, et il importe de «rétablir [son] prestige, la confiance des citoyens dans les institutions, le respect pour la dignité des Forces armées et l'autorité des Forces de sécurité » qui sont «les piliers fondamentaux de notre État démocratique». Santana Lopes affirme que «l’État, comme entité politique qui structure la communauté nationale, est, sans aucun doute, l'instrument de la promotion des intérêts de tous » et lui attribue pour fonctions « d'être un agent actif dans la création des conditions de la justice sociale» et de veiller à «la nécessaire efficacité des services publics». Mais il insiste également sur la place «centrale» des «personnes» qui «constituent une valeur antérieure à la société et à l’État ». Sócrates I prononce quant à lui un vibrant plaidoyer en faveur de l'action de l'État:

Uma economia dinâmica e uma sociedade mais justa não dispensam serviços públicos de qualidade, nem podem prescindir de um Estado com políticas activas de crescimento, de emprego, de redução das desigualdades sociais, mas também de um Estado empenhado nas novas áreas do ambiente, da qualidade urbana e da defesa do consumidor. Só os ricos se podem permitir um Estado pobre e só os poderosos convivem bem com um Estado fraco. 24

24. «Une économie dynamique et une société plus juste ne peuvent se passer de services publics de qualité, ni d'un État qui mène des politiques actives de croissance, d'emploi, de réduction des inégalités sociales, mais qui s'engage aussi dans les nouveaux domaines de l'environnement, 
Sócrates II est plus sobre, mais affirme toutefois le « rôle déterminant» de l'État pour «appuy[er] l'investissement privé et les entreprises» et «stimuler la création d'emploi».

Le point de vue de Coelho est bien différent, puisqu'il s'emploie surtout à définir ce que l'État ne doit pas faire :

Queremos uma sociedade mais aberta, com um Estado que não é um instrumento de obtenção de regalias injustificadas [...] Um Estado que não desista do combate à corrupção. Um Estado que articula e realiza o interesse comum. Um Estado que ajuda a sociedade a florescer e não a sufocá-la. Um Estado que não intimida a criatividade empresarial, nem a inovação. [...]

Queremos um Estado mais pequeno, mais ágil e mais forte, por um lado, e uma sociedade mais livre, mais autónoma e mais próspera, por outro. 25

On est ici face à un cas très net de dialogisme interdiscursif (Bres, Nowakowska, 2006), la critique de Coelho répondant mot pour mot à l'apologie de Sócrates. L'État est vu comme un ennemi potentiel de la société dont il menace le caractère démocratique en détournant des «ressources publiques» au service d' "intérêts privés».

Les emplois de démocratique et démocratie sont eux aussi intéressants mais moins clivants. Ces mots servent tout d'abord à décrire le fonctionnement des institutions portugaises (BA, LO), le Portugal lui-même étant appelé « notre (jeune) démocratie » (SO et $\mathrm{CO}$ ). La démocratie comme régime politique donne une légitimité au gouvernement et au président de la République qui l'investit (SO 2005). Mais elle désigne aussi des principes d'action. Alors qu'il sera très vite accusé d'autoritarisme, Santana Lopes est celui qui emploie le plus le mot et qui insiste sur la «fidélité totale» du gouvernement aux valeurs démocratiques qu'il énumère au fil du discours : responsabilité devant le peuple à qui il faudra rendre des comptes, État au service de tous, "sentiment de justice et de solidarité », sens du dialogue. Sócrates insiste sur le fait que «personne [...] ne sera exclu du processus démocratique, du droit à participer et du droit à être entendu » et il ajoute :

Este Governo governará com sentido nacional e conta com todos, porque sabemos bem que o poder que vamos exercer não é nosso - esse poder é dos cidadãos que queremos representar. ${ }^{26}$ (SO 2005)

de la qualité urbaine et de la défense du consommateur. Seuls les riches peuvent se permettre un État pauvre et seuls les puissants apprécient un État faible.»

25. «Nous voulons une société plus ouverte avec un État qui n'est pas un outil pour obtenir des privilèges injustifiés [...] Un État qui n'abandonne pas le combat contre la corruption. Un État qui articule et met en œuvre l'intérêt commun. Un État qui aide la société à s'épanouir au lieu de l'étouffer. Un État qui n'intimide pas la créativité des entrepreneurs ni l'innovation. [...] Nous voulons un État plus petit, plus agile et plus fort, d'un côté, et une société plus libre, plus autonome et plus prospère, de l'autre.»

26. «Ce gouvernement aura le sens de la nation et s'appuiera sur tout le monde, parce que nous savons bien que le pouvoir que nous allons exercer ne nous appartient pas - il appartient aux citoyens que nous voulons représenter. » 
Il réitère au début de son discours de 2009 l'idée que le gouvernement «en démocratie » est au service de tous. Coelho insiste, lui, sur le fait que «les institutions gouvernementales» doivent se soumettre "au contrôle (escrutínio) démocratique » et être prêtes à «rendre des comptes». La nécessité pour le gouvernement de défendre l'intérêt général est affirmée par tous les locuteurs, mais plus polémiquement, Coelho déclare qu'il s'emploiera à « fortifie[r] l'État devant l'intrusion des intérêts privés ou des clientèles de partis ou de corporations ». Il s'appuie par ailleurs sur la valeur argumentative intrinsèque du mot démocratie (De Chanay, Rémi-Giraud, 2007) pour justifier son programme : "Avec un pays plus ouvert nous aurons une société plus démocratique.»

Le mot société fait l'objet d'emplois très contrastés entre le début et la fin de la décennie. Quasiment absent au début, il devient significativement présent chez Sócrates II et surtout Coelho, alors que les mots peuple et citoyens, qui visent les Portugais en tant qu'ils sont dotés d'un pouvoir politique, disparaissent. Sócrates associe la société à l'économie dans l'effort de modernisation envisagé et se propose de la rendre «plus juste». Quant à Coelho, nous avons vu qu'il oppose la société à l'État qui doit l'aider «à s'épanouir » et non pas «l'étouffer». Malgré ces différences intéressantes, tous les Premiers ministres partagent l'idée qu'une responsabilité morale les lie au pays : leur place à la tête de l'État résulte d'un contrat de confiance qu'ils s'efforcent d'honorer en construisant une image d'eux-mêmes qui les rende dignes de cette confiance. II m'est impossible, dans les limites du présent article, de rendre compte de façon détaillée de la façon dont ils construisent leur ethos dans le discours. On voit bien, lorsqu'on observe les discours sous cet angle, qu'en revendiquant fermeté et courage, en anticipant sur des critiques éventuelles ou en s'opposant à leurs prédécesseurs au nom du réalisme ou de la volonté, ils s'efforcent d'obtenir la confiance du peuple et d'acquérir la crédibilité dont Patrick Charaudeau (2005) fait un des éléments essentiels du politique. Ils mettent aussi en œuvre une éloquence traditionnelle, basée notamment sur les anaphores rhétoriques, qui peut produire un effet de conviction et de détermination propre à rassurer l'auditoire mais qui peut aussi apparaitre comme creuse si elle ne semble pas en prise sur la situation.

Cette étude montre, contrairement à ce qu'on aurait peut-être attendu, que les différences droite-gauche continuent à marquer les choix lexicaux, qu'il s'agisse des relations entre le pays et les citoyens, de la conception de l'État, de la formulation des priorités ou de l'usage du mot démocratie. Mais elle montre aussi de fortes convergences dans la construction de l'image du Portugal, petit pays mais doté d'un rayonnement international important, atlantique mais ancré en Europe. En ce qui concerne la crise économique, les phénomènes d'euphémisation sont nets, et d'autant plus marqués que les difficultés s'approfondissent. Le vocabulaire est certes globalement plus psychologique et 
moral à droite, plus économique et révélateur de la croyance dans les pouvoirs de l'innovation à gauche, mais la schématisation proposée est identique : le pays connaît des difficultés mais il a les ressources pour les surmonter si le gouvernement sait l'orienter dans la bonne direction et si tous participent à l'effort commun. Ces fortes similitudes risquent d'affaiblir le pouvoir de persuasion de ces discours qui peuvent paraitre trop répétitifs. Les buts assignés (restaurer la compétitivité et la croissance, créer des emplois) semblent irréalistes ou trop généraux et l'analyse des causes s'en tient aux chaînons ultimes (la concurrence extérieure, le déficit budgétaire, le poids de la dette) sans remonter plus loin. Le financement des objectifs n'est pas précisé. Le peuple ou le pays sont vus comme des entités homogènes que ne traversent ni conflits d'intérêts, ni clivages sociaux. L'unanimité sur le projet européen, dont on sait combien il conditionne les politiques, laisse douter de la réelle marge de manœuvre de Sócrates, malgré un discours clairement en faveur d'une politique d'investissement orientée vers l'innovation et la réduction des inégalités. Le souci des Premiers ministres de droite de ne laisser personne sur le bord du chemin peut quant à lui se heurter au scepticisme des citoyens qui constatent le creusement des inégalités et l'accès de plus en plus difficile aux soins de santé ou à d'autres biens essentiels. La construction de l'ethos peut alors apparaitre à l'auditoire comme une manœuvre rhétorique destinée à masquer une impuissance réelle à orienter les événements et à agir sur le destin du pays. La détermination et la responsabilité affichées, le positionnement polémique face aux adversaires peuvent-ils se substituer à une véritable action transformatrice qui seule permettrait aux citoyens de reprendre intérêt à la politique?

\section{Sources des discours}

Discours de José Manuel Barroso : obtenu par courrier au siège du Parti social démocrate. Discours de Pedro Santana Lopes : obtenu par courrier au siège du Parti social démocrate. Discours de José Sócrates de 2005 : http://www.publico.pt/politica/noticia/discursode-jose-socrates-na-tomada-de-posse-do-xvii-governo-constitucional-1218111 (consulté le 8 décembre 2012).

Discours de Pedro Passos Coelho de 2011 :

http://www.portugal.gov.pt/media/443405/20110621_pm_int_posse.pdf (consulté le 5 décembre 2012).

\section{Références}

BAсот Paul, 1996, «Des mots pour dire la politisation. État, nation, patrie, pays, peuple dans des corpus politiciens français contemporains», Les mots de la nation, S. Rémi-Giraud, P. Rétat éd., Lyon, PUL, p. 41-64. 
BRES Jacques, NowAKovsKa Aleksandra, 2006, «Dialogisme. Du principe à la matérialité discursive", Le sens et ses voix. Dialogisme et polyphonie en langue et en discours, L. Perrin éd., Recherches linguistiques, n² 28, Metz, Université de Metz.

CharaUdeau Patrick, 2005, Le discours politique. Les masques du pouvoir, Paris, Vuibert.

De ChanAY Hugues, RÉMI-GIRAud Sylvianne, 2007, "Démocratie et ses dérivés. De la dénomination à l'argument sans réplique?», Mots. Les langages du politique, n०83, p. 81-98.

GrIZE Jean-Blaise, 1990, Logique et langage, Paris-Gap, Ophrys.

JALALI Carlos, 2007, Partidos e democracia em Portugal 1974-2005, Lisbonne, Imprensa de Ciências Sociais.

LISI Marco, 2011, Os partidos políticos em Portugal, Coimbra, Almedina.

RÉMI-GIRAUd Sylvianne, 1996, "Le micro-champ lexical français : peuple, nation, État, pays, patrie», Les mots de la nation, S. Rémi-Giraud, P. Rétat éd., Lyon, PUL, p.19-39.

TOURNIER Michel, 1996, "Français à l'extrême droite, un mot habité », Les mots de la nation, S. Rémi-Giraud, P. Rétat éd., Lyon, PUL, p. 65-81. 


\section{Annexe}

Tableau 1. Désignations du Portugal et de ses partenaires

\begin{tabular}{|c|c|c|c|c|c|c|}
\hline & $\begin{array}{c}\text { Durão } \\
\text { Barroso } \\
1428 \text { mots }\end{array}$ & $\begin{array}{c}\text { Santana } \\
\text { Lopes } \\
3970 \text { mots }\end{array}$ & $\begin{array}{l}\text { Sócrates } \\
2005 \\
2631 \text { mots }\end{array}$ & $\begin{array}{l}\text { Sócrates } \\
2009 \\
1849 \text { mots }\end{array}$ & $\begin{array}{c}\text { Passos } \\
\text { Coelho } \\
2265 \text { mots }\end{array}$ & Total \\
\hline Portugal & $16(1,1 \%)$ & $16(\square, 4 \%)$ & $12(\square, 4 \%)$ & $5(\square, 2 \%)$ & $1 \square(\square, 4 \%)$ & 59 \\
\hline $\begin{array}{l}\text { o País / } \\
\text { nosso País }\end{array}$ & $9(\square, 6 \%)$ & $18(\square, 4 \%)$ & $12(\square, 4 \%)$ & $14(\square, 7$ \%) & $8(\square, 3 \%)$ & 51 \\
\hline a pátria & 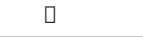 & $\square$ & ( & 2 & ( & 2 \\
\hline $\begin{array}{l}\text { patriótico / } \\
\text { patriotismo }\end{array}$ & 1 & 1 & Q & Q & Q & 2 \\
\hline a nação & 1 & 2 & ( & $\square$ & 1 & 4 \\
\hline nacional $^{\mathrm{a}}$ & 3 & 5 & 5 & 2 & $3^{b}$ & 18 \\
\hline Europa, europeu & $1 \square(\square, 6 \%)$ & $22(\square, 5 \%)$ & $6(\square, 2 \%)$ & 2 & $11(\square, 4 \%)$ & 51 \\
\hline $\begin{array}{l}\text { PLOP/países } \\
\text { lusófonos }\end{array}$ & 1 & 4 & 1 & Q & 1 & 7 \\
\hline o povo & $3^{c}$ & 8 & 5 & 口 & 2 & 18 \\
\hline os Portugueses ${ }^{d}$ & $4(\square, 2 \%)$ & $9(\square, 2 \%)$ & $14(\square, 3 \%)$ & $1 \square(\square, 5 \%)$ & $7(\square, 3 \%)$ & 44 \\
\hline os cidadãos & 1 & $5^{e}$ & 5 & 2 & 2 & 15 \\
\hline
\end{tabular}

a. Les collocations Institut national des statistiques et Service national de santé ne sont pas comptées.

b. Une des trois occurrences est incluse dans le syntagme Programme national d'épargne.

c. Une des occurrences réfère au peuple d'Angola.

d. J'ai retenu les occurrences du syntagme les Portugais et celles du syntagme le peuple portugais, à l'exclusion de syntagmes tels que l'économie portugaise, la société portugaise, la dette externe portugaise, la culture portugaise.

e. Deux occurrences sur les cinq renvoient aux citoyens européens. 
Tableau 2. Dénominations de la crise et des solutions envisagées

\begin{tabular}{|c|c|c|c|c|c|c|}
\hline & $\begin{array}{c}\text { Durão } \\
\text { Barroso } \\
1428 \text { mots }\end{array}$ & $\begin{array}{c}\begin{array}{c}\text { Santana } \\
\text { Lopes }\end{array} \\
3970 \text { mots }\end{array}$ & $\begin{array}{c}\text { Sócrates } \\
2005 \\
2631 \text { mots }\end{array}$ & $\begin{array}{c}\text { Sócrates } \\
2009 \\
1849 \text { mots }\end{array}$ & $\begin{array}{c}\text { Passos } \\
\text { Coelho } \\
2265 \text { mots }\end{array}$ & Total \\
\hline crise & 5 & 1 & $\mathrm{Q}$ & 6 & 3 & 15 \\
\hline dificuldade(s) & 1 & 6 & 2 & 1 & 3 & 13 \\
\hline difícil/difíceis & 2 & 2 & 3 & 1 & 2 & $1 \mathrm{~L}$ \\
\hline problema(s) & 2 & 6 & 3 & 1 & 6 & 18 \\
\hline bloqueio(s) & $\square$ & $\mathrm{Q}$ & 1 & $\square$ & 2 & 3 \\
\hline atraso(s) & 1 & 1 & 1 & $\mathrm{Q}$ & $\mathrm{Q}$ & 3 \\
\hline $\operatorname{des} a f i o(s)^{\mathrm{a}}$ & Q & 5 & 5 & 6 & 1 & 17 \\
\hline futuro & 2 & $\square$ & 5 & 4 & 1 & 12 \\
\hline esperança & 3 & 2 & $\square$ & $\mathrm{Q}$ & $\mathrm{Q}$ & 5 \\
\hline esforço(s) & 2 & 10 & 1 & 3 & 4 & $2 \square$ \\
\hline reforma(s) & $\square$ & Q & $\square$ & 3 & $a^{b}$ & 3 \\
\hline progresso & 1 & 4 & 2 & 2 & $\square$ & 9 \\
\hline mudança(s) & 2 & $\square$ & 3 & 6 & 10 & 21 \\
\hline $\begin{array}{l}\text { moderno/ } \\
\text { modernização }\end{array}$ & ० & 2 & 2 & 15 & Q & 19 \\
\hline inovação/novador & $\square$ & 5 & 5 & 2 & 2 & 14 \\
\hline $\begin{array}{l}\text { aberto/ } \\
\text { abertura }^{c}\end{array}$ & 1 & 3 & 1 & 2 & 20 & 24 \\
\hline
\end{tabular}

a. bloqueio : «blocage», atraso : « retard », desafio : « défi ».

b. On trouve une fois l'expression un esprit réformiste

c. Je n'ai retenu que les cas où l'ouverture était présentée comme un remède ou un projet, j'ai donc exclu par exemple les constats sur l'économie mondiale de plus en plus ouverte.

Tableau 3. Objectifs assignés à l'action gouvernementale

\begin{tabular}{|c|c|c|c|c|}
\hline Durão Barroso & Santana Lopes & Sócrates 2005 & Sócrates 2009 & Passos Coelho \\
\hline $\begin{array}{l}\text { 1. Assainissement } \\
\text { budgétairea } \\
\text { 2. Correction des } \\
\text { déséquilibres } \\
\text { macroécono- } \\
\text { miques } \\
\text { 3. Justice sociale } \\
\text { 4. Dignité de l'État } \\
\text { 5. Projection du } \\
\text { Portugal dans le } \\
\text { monde }\end{array}$ & $\begin{array}{l}\text { 1. Faciliter l'inves- } \\
\text { tissement public, } \\
\text { privé, national et } \\
\text { étranger } \\
\text { 2. Restaurer la com- } \\
\text { pétitivité des pro- } \\
\text { duits portugais } \\
\text { 3. Développer le } \\
\text { tourisme } \\
\text { 4. Développer la } \\
\text { recherche et l'in- } \\
\text { novation }\end{array}$ & $\begin{array}{l}\text { 1. Vaincre les causes } \\
\text { structurelles du } \\
\text { retard portugais: } \\
\text { connaissance et } \\
\text { innovation } \\
\text { 2. Retrouver la crois- } \\
\text { sance et com- } \\
\text { battre le chômage } \\
\text { 3. Réduire les inéga- } \\
\text { lités sociales et la } \\
\text { pauvreté }\end{array}$ & $\begin{array}{l}\text { 1. Rétablir la crois- } \\
\text { sance et l'emploi } \\
\text { 2. Moderniser } \\
\text { l'économie et la } \\
\text { société (innova- } \\
\text { tion) } \\
\text { 3. Justice sociale } \\
\text { et réduction des } \\
\text { inégalités }\end{array}$ & $\begin{array}{l}\text { 1. Stabiliser les } \\
\text { finances et } \\
\text { réduire la dette } \\
\text { 2. Secourir les plus } \\
\text { vulnérables } \\
\text { 3. Développer la } \\
\text { croissance, la } \\
\text { compétitivité et } \\
\text { l'emploi dans le } \\
\text { secteur des biens } \\
\text { marchands }\end{array}$ \\
\hline
\end{tabular}

a. Tous les termes du tableau sont la traduction française de syntagmes extraits des discours eux-mêmes. 
Tableau 4 : fréquences des lexèmes gouvernement, État, société et démocratie

\begin{tabular}{lcccccc}
\hline & $\begin{array}{c}\text { Durão } \\
\text { Barroso }\end{array}$ & $\begin{array}{c}\text { Santana } \\
\text { Lopes }\end{array}$ & Sócrates I & Sócrates II & $\begin{array}{c}\text { Passos } \\
\text { Coelho }\end{array}$ & Total \\
\hline $\begin{array}{c}19 \\
\text { Governo }\end{array}$ & $\begin{array}{c}29 \\
\text { (douv. } \\
\text { précédent) } \\
(1,3 \%)\end{array}$ & $\begin{array}{c}\text { (dont } \\
\text { antérieur) } \\
(\square, 7 \%)\end{array}$ & $28(1 \%)$ & $2 \square(1 \%)$ & $13(\square, 5 \%)$ & $1 \square 9$ \\
\hline Estado & $5(\square, 3 \%)$ & $13(\square, 3 \%)$ & $7(\square, 2 \%)$ & $4(\square, 2 \%)$ & $12(\square, 5 \%)$ & 41 \\
\hline sociedade & 1 & 1 & 2 & $5(\square, 2 \%)$ & $1 \square(\square, 4 \%)$ & 19 \\
\hline interesse geral & 2 & $\square \mathrm{a}$ & 2 & 4 & $4 \mathrm{~b}$ & 8 \\
\hline público ${ }^{c}$ & 1 & 3 & 4 & 7 & 4 & 19 \\
\hline $\begin{array}{l}\text { democracia, } \\
\text { democrático }\end{array}$ & 2 & $1 \square$ & 5 & 3 & 6 & 26 \\
\hline
\end{tabular}

a. On trouve intérêt national, intérêt de tous, intérêt du pays, opposés aux intérêts égoïstes ou clientélistes.

b. On trouve une fois l'intérêt commun et deux fois les intérêts privés connotés négativement.

c. J'ai exclu finances publiques et comptes publics (6 occurrences pour BA, 2 pour LO, 5 pour SO I, 3 pour (O). Les syntagmes concernés sont Administration publique, cause publique, Sécurité sociale publique, réponse publique, geste public, biens publics, services publics, investissement public, contrôle public, ressources publiques, partenariat public-privé. 